\title{
THE REMARK ABOUT AN ANALOGY BETWEEN MAGNETOCALORIC BEHAVIOUR OF TYPE II SUPERCONDUCTORS AND FERROMAGNETIC MATERIALS
}

\author{
Z. KALVA AND P. MÁNEK \\ Center for Theoretical Study, Charles University and the Academy of Sciences \\ Jilska 1, 11000 Praha 1, Czech Republic
}

The analogy between magnetocaloric behaviour of type II superconductors and anisotropic ferromagnetic materials is discussed. Special attention is devoted to the influence of anisotropy on the negative magnetocaloric effect near phase transitions.

PACS numbers: $75.30 . \mathrm{Sg}, 74.60 . \mathrm{Ge}$

\section{Introduction}

This paper concerns mainly the magnetocaloric behaviour of ferromagnetic materials, which have a phonon system and a magnon system mutually interacting. Our earlier calculations $[1,2]$ described the thermodynamic dissipation of energy evoked by an increase in the external magnetic field in an adiabatically isolated system. The change of sign of the magnetocaloric effect was deduced in the vicinity of the magnetic phase transitions $[1,3,4]$. The increase in magnetic field to the critical value $H^{\mathrm{c}}$ in the direction perpendicular to the easy anisotropic axis of a ferromagnetic material causes the magnetization to turn and saturate in the direction of the external magnetic field and it can also result in a decrease in temperature (the sign of magnetocaloric effect becomes negative). In Ref. [2] it was shown that a similar behaviour can be expected also in the case of superconductors of type II in the mixed state. A change of sign of the magnetocaloric effect in superconductors can be expected near the transition from a superconducting state into a state of normal conductivity. In this case the transfer of energy between collective excitations of crystalline and vortex lattices will be responsible for the negative magnetocaloric effect.

\section{Calculations of the magnetocaloric effect}

One of the open questions in our calculations is the influence of anisotropy. The magnetic anisotropy creates different conditions for the dissipation of energy 
below and above the magnetic phase transition. The anisotropy of the atomic lattice which forms the basis for phonon-type excitations can also be important and this remark is the preliminary attempt to study the influence of non-magnetic anisotropy on the magnetocaloric effect. We turned our attention to the variation of elastic constants of the material and studied the dependence of the magnetocaloric effect on the ratio of the longitudinal and transversal speed of elastic waves, which is a function of Poisson's elastic constant. We have chosen here this parameter for practical reasons. Our formulae for the energies of collective excitations (we shall call them quasimagnons and quasiphonons) depend on the ratio of transversal and longitudinal velocity of sound. We posed the following question: how does the magnetocaloric effect depend on the elastic constants? A dependence was found by the iterative numerical modelling, is given in Fig. 1 and is described in the following text.

In the vicinity of the magnetic phase transition we forecasted in $[1,2]$ an anomalous behaviour of quasiphonons. For the specific directions the energy of quasiphonons approaches zero, which could be understood as an anomalous decrease in the speed of sound waves. A similar effect could be possible also for the vortex lattice excitations in superconducting materials. Interactions of sound waves with the vortex lattice is now an interesting research field and there is a hope that it can contribute to understanding of the superconductivity in new materials [5]. From this point of view it is therefore also interesting to study the analogy between magnetocaloric behaviour of superconductors and classical magnetic materials.

The decrease in quasiparticle energy leads to an anomalously higher population of corresponding quasiparticles which can influence the magnetocaloric behaviour of the whole system. The population of bosons will grow substantially with decreasing energy. The energy $E$ accumulated in collective quasiparticle-like excitations is

$$
\lim _{\omega \rightarrow 0} E=\sum_{k} \frac{\omega_{k}}{\mathrm{e}^{\omega_{k} / k_{\mathrm{B}} T}-1} \sim k_{\mathrm{B}} T
$$

(where $\omega$ is the energy of quasiphonon with wave vector $k, k_{\mathrm{B}}$ is Boltzmann's constant and $T$ is a temperature of crystalline lattice) and it will be still bigger than in the case of "normal" non-zero quasiparticle energies.

The formulae for the quasiparticle energies and the model Hamiltonian can be found in [1] and [2] (but without explanatory remarks). We made an approximative diagonalisation of the Hamiltonian. (Calculations with better Hamiltonian are now planned in collaboration with experts from Wrocław.) The diagonalisation was done in two steps. We found separately operators for magnons and phonons; for magnons from the magnetic part of the Hamiltonian and for phonons from the term corresponding to a cubic isotropic material. The second step of the diagonalisation was done by including an explicit term for magnetoelastic interaction in the Hamiltonian. We used the simplest formula which combines together the elasticity of a crystalline lattice and magnetic moments $M$

$$
H^{\text {magnetoelastic }}=g M_{i} M_{k} u_{i k},
$$

where $g$ is magnetoelastic constant and $u_{i k}$ is the tensor of deformation. 
Magnon and phonon operators obtained from the first step of the diagonalisation procedure were substituted in the magnetoelastic term and the resulting Hamiltonian was approximately diagonalised by the standard procedure (Bogolyubov transformation). The condition of solubility of the system of 8 equations (the secular equation) made it possible to find analytically the energies of quasimagnons and quasiphonons. A general solution was out of reach, but we were able to find approximative solutions for the special directions and relatively weak interaction between magnetic and elastic system (the constant of uniaxial magnetic anisotropy $\beta$ should be significantly larger than the constant $g$ in the magnetoelastic term). The "individuality" of phonons and the "individuality" of magnons is in this case preserved and we looked for the solution of the secular equation in the phonon-like and magnon-like shapes. For quasiphonons $\omega=v k$ (the function $v$ has to be found and now it depends also on the external magnetic field), and for quasimagnons $\varepsilon=\varepsilon_{0}(k)^{2}$.

The formulae for the quasimagnon and quasiphonon energies are given in [2] and we shall reproduce here only the corrected term for two quasiphonon branches which contains in [2] the small printing error. The energy of one branch of quasiphonons tends for the $z$ and $x$ directions to zero in the neighbourhood of our magnetic phase transition and it has important consequences for the magnetocaloric effect

$$
\begin{aligned}
& \omega_{1,2}^{2}=\frac{1}{2}\left[c_{t}^{2}+c_{l}^{2}-\phi c_{t}^{2}\left(k_{x}^{2}+k_{z}^{2}\right)\right] \\
& \pm \sqrt{\frac{1}{4}\left[c_{t}^{2}+c_{l}^{2}-\phi c_{t}^{2}\left(k_{x}^{2}+k_{z}^{2}\right)\right]^{2}-c_{t}^{2} c_{l}^{2}\left[1-\phi\left(k_{x}^{2}+k_{z}^{2}\right)\right]+4 \phi c_{t}^{2}\left(c_{t}^{2}-c_{l}^{2}\right)\left(k_{x}^{2} k_{z}^{2}\right)}, \\
& \phi=\frac{\Delta l}{\Delta l+\Delta h}\left(k_{x}^{2}+k_{z}^{2}\right)
\end{aligned}
$$

$\left(c_{t}, c_{l}\right.$ are transverse and longitudinal sound speeds). The magnetocaloric effect was calculated using the standard formula for entropy for the case of an adiabatically isolated sample and all modes of quasiphonons and quasimagnons. The formulae for energies of quasiparticles are of course valid only for small wave vectors, but the influence of larger wave vectors is negligible in the case that the energy of quasiparticle is considerably smaller than $k_{\mathrm{B}} T$ (in our calculations $T \approx 20 \mathrm{~K}$ ).

\section{Results}

The example of a dependence of the magnetocaloric effect on the variation of the ratio $c_{l} / c_{t}$ is shown in Fig. 1. A similar dependence of a lattice temperature on an external magnetic field could be expected also in the superconductors of the second type near the transition to the normal conductivity - enforced by the growing magnetic field - because the energy of collective excitations of the vortex lattice tends to zero. Energy is transferred from the thermal vibrations into collective excitations of the vortex lattice and the sign of the magnetocaloric effect is going to be negative. In Ref. [2] are presented some preliminary calculations of these phenomena. 


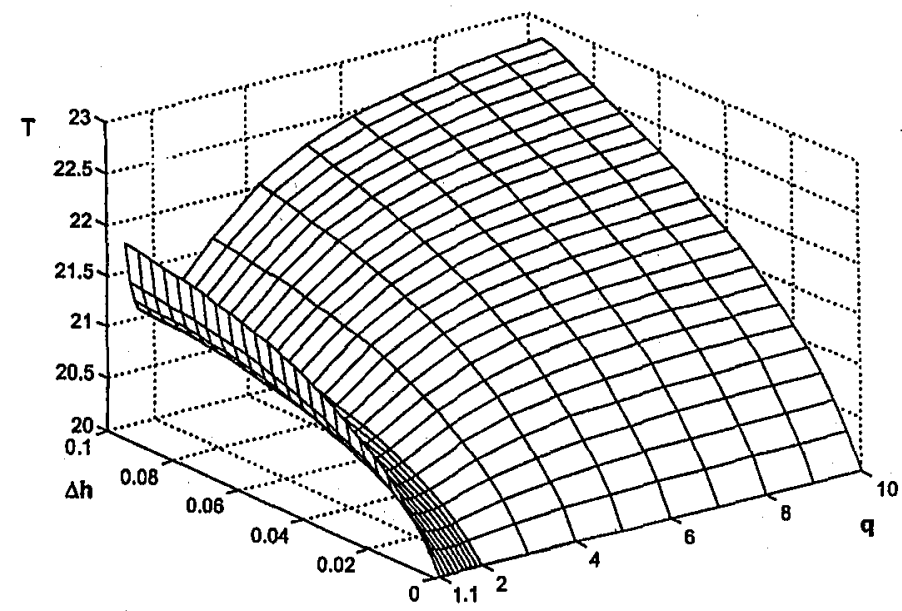

Fig. 1. Magnetocaloric effect. $\Delta h$ is the external magnetic field in relative units $(\Delta h=0$ is in the phase transition $H=H^{\mathrm{c}}$ ). The lattice temperature $T$ goes down to $20 \mathrm{~K}$. $q=c_{\text {longitudinal }} / c_{\text {transversal }}$.

The curves in Fig. 1 show that the maximal decrease in temperature can be expected for small $q$. The minimal change of lattice temperature is for $q \approx \sqrt{2}$. From the values of elastic constants in real physical materials ( $q_{\text {tungsten }}=1.57$, $q_{\text {steel }}=1.8, q_{\text {lead }}=3.3$ ) is possible to see that lower limit for parameter $q$ is a bit greater than $\sqrt{2}$. Figure 1 further shows that the dependence of the magnetocaloric effect on the parameter $q$ is nonlinear.

\section{Concluding remarks}

The dependence of a size and sign of the magnetocaloric effect on the non-magnetic elastic characteristic of materials presented in this remark is only informative and preliminary and we hope that the planned collaboration with physicists from Wrocław will provide results for real materials. We believe that such study of magnetocaloric effect is an effective tool for better understanding of dissipative processes especially in new superconducting materials. We believe that the quasiparticle approach of second quantisation, used also in our work, is the proper way to go.

\section{Acknowledgment}

The authors acknowledges with thanks the support by grant GAUK 163/97 B Fyz from Charles University.

\section{References}

[1] Z. Kalva, Acta Physica Slovaca 46, 5 (1996).

[2] T. Svobodny, Z. Kalva, Phys. Status Solidi B 208, 187 (1998).

[3] L. Biegala, Z. Kalva, I. Veltrusky, Acta Phys. Pol. A 55, 687 (1979).

[4] Z. Kalva, I. Veltrusky, L. Biegala, G. Kozlowski, Phys. Lett. A 57, 75 (1976).

[5] D. Domingues, L. Bulaevskij, B. Ivlev, M. Maley, A.R. Bishop, Phys. Rev. Lett. 74, 2579 (1995). 